\title{
Angular and Frequency Correlation for Sea-Ice Thickness Retrieval
}

\author{
Ziad A. Hussein ${ }^{1}$, Yasuo Kuga ${ }^{2}$, Akira Ishimaru ${ }^{3}$, \\ Sermsak Jaruwatanadilok ${ }^{4}$, Kyung Pak ${ }^{5}$ \\ 1,5 Jet Propulsion Laboratory \\ California Institute of Technology \\ Pasadena, CA 91109. email: ziad.a.hussein@jpl.nasa.gov \\ ${ }^{2,3,4}$ University of Washington, Seattle \\ Department of Electrical Engineering \\ Seattle, WA 98195
}

\begin{abstract}
A combined spatial and frequency domain interferometer or angular and frequency correlation (ACF/FCF) between two radar beams in the VHF-band is applied for the direct measurement of sea-ice thickness. This measurement is critical because the thickness of sea ice within the polar region indicates the state of ocean circulation and the associated air-sea heat exchange, which profoundly affects the global heat balance and ocean thermohaline circulation. This new instrument technologycryospheric advanced sensor (CAS) - can measure sea-ice thickness, filling a critical gap in measuring the polar region. In this paper, we present the algorithm development and demonstration by simulations of estimating the height of the sea-ice that led to the robust design of CAS interferometric system. Sea-ice thickness is derived from the interferometric phase of the ACF/FCF function of two VHF-bandscattered returns of two radar waves that have different frequencies, incident angles, and observation angles. The inversion calculation to estimate the ice thickness is based on several methods, gradientdescent (GD), least-square (LSQ) method, and genetic algorithm (GA). Compared with a GD method, and LSQ method, GA does not require the knowledge of the derivative of the $\mathrm{ACF} / \mathrm{FCF}$ function. Good agreement is shown with GD and LSQ results, when a single unknown variable - sea-ice thickness - is to be determined. To support the inversion calculations and analysis, we developed an analytical model. The analytical model used to formulate the $\mathrm{ACF} / \mathrm{FCF}$ function depends on the age of the ice being measured. The analytical model for first-year ice is based on the small perturbation method (presented here) and, for multiyear ice, the Kirchhoff approximation (presented in accompanying paper by the authors).
\end{abstract}

Index Terms - Correlation function, sea ice thickness, rough surface scattering.

\section{INTRODUCTION}

$\mathrm{S}_{\mathrm{oc}}^{\mathrm{E}}$ EA ice Sea ice thickness is an indicator of the state of ocean circulation and associated air-sea heat exchange within the Polar Regions, which can have profound impacts on global heat balance and ocean thermohaline circulation. Developing a means of determining sea ice thickness cover on broad, synoptic-scales at regular intervals would fill one of the highest priority measurement gaps in the polar-regions. For a given low frequency of operation, available radar bandwidth is limited for a spaceborne sensor. This limits the techniques (e.g. radar sounder) that can be applied to the direct measurement of sea-ice thickness. In this paper, we present sea-ice thickness estimation based on combined spatial and frequency domain interferometer or angular and frequency correlations (ACF/FCF) between two radar waves at different frequencies, incident angles and observations angles. This approach allows alleviating the restriction of very wide radar bandwidth as in radar sounder, and has an advantage to differentiate surface scattering (at the air-snow, snow-ice, and ice-water interfaces) and volume scattering (due to brine inclusions and air bubbles within the ice). This approach has an advantage to exploit the difference in the effect of correlation properties of surface (strong correlation along a line- memory line) and volume scattering (strong correlation at specific points -memory dots). Consequently, the interferometric phase of the angular and frequency correlation function of two scattered waves is used to derive the corresponding sea ice thickness, and offers a means to develop a robust interferometric system.

Our forward calculations use four-layer sea-ice model (air, snow, sea-ice, and seawater). We have developed analytical and numerical models. The analytical models ACF/FCF function formulation is based on small perturbation method (useful for first-year ice) and Kichhoff approximation (useful for multi-year ice). We used the ACF/FCF functions to gain insight into its coherence amplitude and phase sensitivity with ice depth, surface layers roughness, and volume scattering suppression, and guide in the instrument design.

We present the sea-ice inversion calculation of the CAS based on a Genetic Algorithm (GA), and is compared to Gradient Descent (GD) method, Least Square Method (LSQ). We also present snow depth effect, and the uncertainty associated with the knowledge of the dielectric constant of the sea-ice brine inclusion on sea-ice thickness estimation.

\section{SEA ICE MODEL}

Sea ice is a highly inhomogeneous medium consisting of lossy background ice with trapped air bubbles and brine inclusions. Furthermore, the surface is covered by snow and its compositions vary substantially over time and location. At low frequency such as VHF radar, the detailed structures of sea ice may not be important, and we can model the sea ice as a four-layer medium which consists of air, snow, ice, and 
TABLE I

SEA ICE MODEL PARAMETERS

\begin{tabular}{lccc}
\hline \hline & Relative dielectric constant & Layer thickness (m) & rms height (cm) \\
& $\varepsilon_{2}=3.15+j 0.001$ & $>0.08$ & 0.024 \\
$\begin{array}{l}\text { Layer 2: snow; } \\
\begin{array}{l}\text { Rough surface 1: air-snow } \\
\text { Rayer 3: ice; }\end{array}\end{array}$ & $\varepsilon_{3}=4.4176+j 0.5107$ & $>2$ & 0.12 \\
$\begin{array}{l}\text { Laygh surface 2: snow-ice } \\
\text { Rough surface 2: snow-ice }\end{array}$ & $\varepsilon_{4}=57.9+j 39.8$ & 24 \\
\hline \hline
\end{tabular}

Note that Layer 1 is air with relative dielectric constant of 1 .

seawater layers as shown in Fig.1. This has three boundaries which can contribute to the total radar return: the air to snow boundary, snow to sea ice boundary, and sea ice to seawater boundary. In addition, the volume scattering due to inclusions in the snow and ice layer may become important if the boundaries are smooth. If the temperature is above freezing, the snow layer may contain a mixture of air and moisture as the background material known as 'wet air'. Based on the published reports [3], [5], we estimated the typical parameters of this four layer structures as shown in Table 1. The EM model presented in this paper is obtained using these values.

To simplify the analysis and demonstrate the effectiveness of the proposed approach, we only present one-dimensional model in this paper. We separate the EM wave propagating in the medium into coherent and incoherent waves. For coherent wave, we use transmission line approach in which the boundaries are assumed to be flat and multiple reflections in snow and sea ice layers are included. Once the down-going and up-going coherent waves are obtained, we can find the incoherent wave induced by the rough surface scattering and volume scattering. The interfaces between layers are very small compared to the wavelength; thus the small perturbation approximation is used for rough surface scattering. The inclusions in both snow and ice layer are also very small compared to the wavelength. Therefore, we can use Rayleigh approximation for volume scattering.

The dielectric constants shown in Table 1 are the result of the Maxwell-Garnett mixing formula given by [2]

$\varepsilon_{\text {eff }}=\varepsilon_{1} \frac{1+2 f y}{1-f y}, y=\frac{\varepsilon_{2}-\varepsilon_{1}}{\varepsilon_{2}+2 \varepsilon_{1}}$

where $\varepsilon_{\text {eff }}$ is the effective dielectric constant, $\varepsilon_{1}$ is the dielectric constant of the background medium, $\varepsilon_{2}$ is the dielectric constant of the inclusions, and $f$ is the fractional volume. The dominant inclusions that strongly affect the dielectric constant value of bulk sea ice are the brine inclusions because it has a large value of dielectric constant in both real and imaginary part. Here we use fractional volume of up to $5 \%$ for brine inclusions.

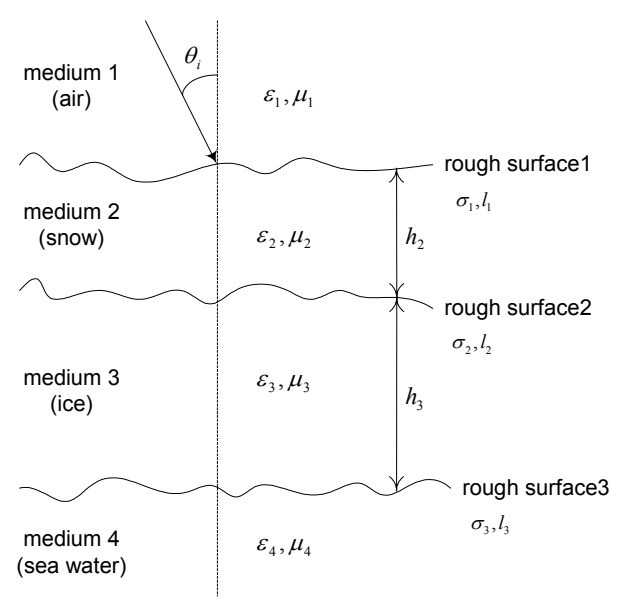

Fig 1. Sea ice model.

\section{A. Coherent component}

The coherent component in this case in the component resulting from multiple reflections within media based on the flat surface interfaces. It is considered a zeroth-order solution to this multilayer media problem. The coherent component can be calculated based on the transmission line model. Let $\psi_{d 1}$ and $\psi_{u 1}$ be the up-going and down-going wave in medium 1, respectively. The coherent up-going and down-going wave in layer 1 (air), 2 (snow), and 3 (ice) can be computed by [2]

$$
\begin{aligned}
& {\left[\begin{array}{l}
V_{1} \\
I_{1}
\end{array}\right]=\left[\begin{array}{cc}
1 & 1 \\
1 / Z_{1} & -1 / Z_{1}
\end{array}\right]\left[\begin{array}{l}
\psi_{d 1} \\
\psi_{u 1}
\end{array}\right]} \\
& {\left[\begin{array}{l}
V_{2} \\
I_{2}
\end{array}\right]=\left[\begin{array}{cc}
\exp \left(-i q_{2} h_{2}\right) & \exp \left(i q_{2} h_{2}\right) \\
\exp \left(-i q_{2} h_{2}\right) / Z_{2} & -\exp \left(i q_{2} h_{2}\right) / Z_{2}
\end{array}\right]\left[\begin{array}{l}
\psi_{d 2} \\
\psi_{u 2}
\end{array}\right]} \\
& {\left[\begin{array}{l}
V_{3} \\
I_{3}
\end{array}\right]=\left[\begin{array}{cc}
\exp \left(-i q_{3} h_{3}\right) & \exp \left(i q_{3} h_{3}\right) \\
\exp \left(-i q_{3} h_{3}\right) / Z_{3} & -\exp \left(i q_{3} h_{3}\right) / Z_{3}
\end{array}\right]\left[\begin{array}{l}
\psi_{d 3} \\
\psi_{u 3}
\end{array}\right]}
\end{aligned}
$$

where

$$
V_{1}=E_{o}\left(1+R_{s}\right), I_{1}=\frac{E_{o}}{Z_{1}}\left(1-R_{s}\right), V_{3}=E_{o} T_{s}, I_{3}=\frac{E_{o} T_{s}}{Z_{4}},
$$


$V_{1}$ and $I_{1}$ are evaluated at air-snow interface, $V_{2}$ and $I_{2}$ are at snow-sea ice interface, and $V_{3}$ and $I_{3}$ are at sea ice-water interface, respectively.

$\left[\begin{array}{l}V_{2} \\ I_{2}\end{array}\right]=\left[\begin{array}{ll}A_{2} & B_{2} \\ C_{2} & D_{2}\end{array}\right]\left[\begin{array}{c}V_{1} \\ I_{1}\end{array}\right]$,

$R_{s}=\frac{A+B / Z_{4}-Z_{1}\left(C+D / Z_{4}\right)}{A+B / Z_{4}+Z_{1}\left(C+D / Z_{4}\right)}$,

$T_{s}=\frac{2}{A+B / Z_{4}-Z_{1}\left(C+D / Z_{4}\right)}$,

$\left[\begin{array}{ll}A & B \\ C & D\end{array}\right]=\left[\begin{array}{ll}A_{2} & B_{2} \\ C_{2} & D_{2}\end{array}\right]\left[\begin{array}{ll}A_{3} & B_{3} \\ C_{3} & D_{3}\end{array}\right]$,

$A_{m}=D_{m}=\cos q_{m} h_{m}$,

$B_{m}=j Z_{m} \sin q_{m} h_{m}, C_{m}=\frac{j \sin q_{m} h_{m}}{Z_{m}}, q_{m}=k \cos \theta_{m}$

The impedance $Z_{m}$ for the $\mathrm{m}^{\text {th }}$ medium is

$Z_{m}=\frac{\omega \mu_{m}}{q_{m}}$ for TE wave, $Z_{m}=\frac{q_{m}}{\omega \varepsilon_{m}}$ for TM wave.

The angle $\theta_{m}$ in each layer can be calculated using Snell's law.

\section{B. Incoherent component-rough surfaces}

We use the small perturbation approximation to calculate the rough surface scattering contribution. Consider the interface between two media with rough surface as shown in Fig. 2.

The incident wave field $\psi_{i}$, the scattered wave field $\psi_{s}$, and the transmitted wave field $\psi_{t}$ are given by

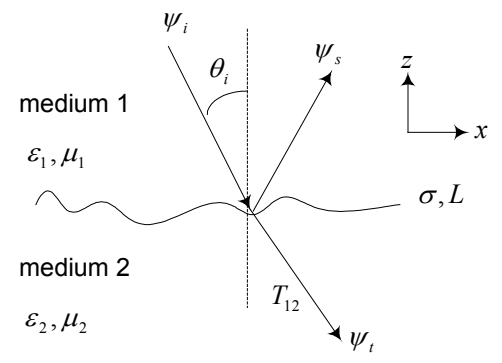

Fig 2. Rough surface geometry.

$\psi_{i}=\frac{1}{2 \pi} \int A\left(K_{i}\right) \exp \left(-i K_{z i} z+i K_{i} x\right) d K_{i}$,

$\psi_{s}=\frac{1}{2 \pi} \int B(K) \exp \left(-i K_{z 1} z+i K x\right) d K$

$\psi_{t}=\frac{1}{2 \pi} \int C(K) \exp \left(-i K_{z 2} z+i K x\right) d K$

where

$K_{z i}=\sqrt{k_{1}^{2}-K_{i}^{2}}, K_{z 1}=\sqrt{k_{1}^{2}-K^{2}}, K_{z 2}=\sqrt{k_{2}^{2}-K^{2}}, K_{i}=k_{1} \sin \theta_{i}$ and $K=k_{o} \sqrt{\varepsilon_{e f f}} \sin \theta$. The function $B(K)$ and are $C(K)$ the transition operators defined as
$B(K)=\int S_{b}\left(K, K_{i}\right) A\left(K_{i}\right) d K_{i}$,

$C(K)=\int S_{c}\left(K, K_{i}\right) A\left(K_{i}\right) d K_{i}$.

We expand $S_{b}$ and $S_{c}$ in scattering order and retain zerothorder and first-order terms.

$$
\begin{aligned}
& S_{b}\left(K, K_{i}\right)=S_{b o}\left(K, K_{i}\right)+S_{b 1}\left(K, K_{i}\right)+\ldots, \\
& S_{c}\left(K, K_{i}\right)=S_{c o}\left(K, K_{i}\right)+S_{c 1}\left(K, K_{i}\right)+\ldots
\end{aligned}
$$

The zeroth-order solution is the solution of the coherent wave problem using the transmission line approach. It can be written as

$S_{b o}\left(K, K_{i}\right)=R\left(K, K_{i}\right) \delta\left(K-K_{i}\right)$,

$S_{c o}\left(K, K_{i}\right)=T\left(K, K_{i}\right) \delta\left(K-K_{i}\right)$.

The first-order solution is given by

$$
\begin{aligned}
& S_{b 1}\left(K, K_{i}\right)=\frac{1}{2 \pi} \frac{\frac{j K_{z 2} A}{\varepsilon_{r}}+B}{\frac{j K_{z 2}}{\varepsilon_{r}}+j K_{z i}} H\left(K-K_{i}\right) \\
& S_{c 1}\left(K, K_{i}\right)=\frac{1}{2 \pi} \frac{B-j K_{z 2} A}{\frac{j K_{z 2}}{\varepsilon_{r}}+i K_{z i}} H\left(K-K_{i}\right)
\end{aligned}
$$

where $\varepsilon_{r}=\varepsilon_{2} / \varepsilon_{1}$,

$A=\left(j K_{z i}-j K_{z 1} R-j K_{z 2} T\right)$

$B=\left(-\left(K_{i} K-k_{2}^{2}\right)(1+R)+\frac{1}{\varepsilon_{r}}\left(K_{i} K-k^{2}\right) T\right)$

$R$ and $T$ is the reflection and transmission coefficient, respectively.

The function $H$ is given by

$H\left(K-K_{i}\right)=\int h(x) \exp \left(-j\left(K-K_{i}\right) x\right) d x$

where $h(x)$ is the height function of the random surface.

We can find the far-field scattered wave is given by

$\psi_{s}\left(K, K_{i}\right)=\frac{k \cos \theta}{(2 \pi k R)^{1 / 2}} \exp \left(-j k R+j \frac{\pi}{4}\right) S\left(K, K_{i}\right)$

Note that $S\left(K, K_{i}\right)$ is a function of $H\left(K, K_{i}\right)$.

For convenience, we write the scattered field $\psi_{s}\left(\theta_{s 1}, \omega_{1}\right)$ as a product of two terms

$\psi_{s}\left(\theta_{s 1}, \omega_{1}\right)=\Theta_{s}\left(\theta_{s 1}, \omega_{1}\right) H\left(K-K_{i}, \omega\right)$.

Now, we have the scattered field formulation from one rough surface. The total scattered waves are combination of rough surface scattering from all the surfaces and volume scattering from inclusions in layers. We further make assumption that these scattering phenomena are uncorrelated. Thus, the correlation of scattered waves is the summation of the correlation of wave from each rough surface and correlation of wave from volume scattering. The total correlation function is given by

$$
\left\langle\psi_{t} \psi_{t}^{*}\right\rangle=\left\langle\psi_{s 1} \psi_{s 1}^{*}\right\rangle+\left\langle\psi_{s 2} \psi_{s 2}^{*}\right\rangle+\left\langle\psi_{s 3} \psi_{s 3}^{*}\right\rangle+\left\langle\psi_{v 1} \psi_{v 1}^{*}\right\rangle+\left\langle\psi_{v 2} \psi_{v 2}^{*}\right\rangle
$$

where s1, s2, and s3 denotes the rough surface 1 (air-snow), rough surface 2 (snow-ice), and rough surface 3 (iceseawater), and v1, v2 denotes the volume scattering from air bubbles and brine inclusions, respectively. 
For rough surface scattering, the angular/frequency correlation function $(\mathrm{ACF} / \mathrm{FCF})$ is in the form

$$
\left\langle\psi_{s}\left(\theta_{s 1}, \omega_{1}\right) \psi_{s}\left(\theta_{s 2}, \omega_{2}\right)^{*}\right\rangle=\left[\Theta_{s}\left(\theta_{s 1}, \omega_{1}\right) \Theta_{s}^{*}\left(\theta_{s 2}, \omega_{2}\right)\right]\left\langle H_{1} H_{2}^{*}\right\rangle
$$

where

$$
\begin{aligned}
& \left\langle H_{1} H_{2}^{*}\right\rangle=\sigma^{2} \pi L_{e q} l \exp \left(-\frac{A_{c}^{2} l^{2}}{4}\right) \exp \left(-\frac{A_{d}^{2} L_{e q}{ }^{2}}{4 \pi}\right), \\
& A_{d}=k_{1} \sin \theta_{s 1}-k_{2} \sin \theta_{s 2}-\left(k_{1} \sin \theta_{i 1}-k_{2} \sin \theta_{i 2}\right), \\
& A_{c}=\frac{1}{2}\left[k_{1}\left(\sin \theta_{s 1}-\sin \theta_{i 1}\right)+k_{2}\left(\sin \theta_{s 1}-\sin \theta_{i 2}\right)\right] .
\end{aligned}
$$

where $\sigma$ is the rms height and $l$ is the correlation length. $L_{e q}$ is the illumination length of the surface which is included in a Gaussian taper function. Although the previous formulation is based on a plane wave, the Gaussian taper function is included in Eq (12) to simulate the realistic case.

The function $\Theta$ can be found using Eq. (7), (9) and (10) as.

$$
\Theta_{s}(\theta, \omega)=\frac{k \cos \theta}{(2 \pi k R)^{1 / 2}} \exp \left(-j k R+j \frac{\pi}{4}\right) \frac{1}{2 \pi} \frac{\frac{j K_{z 2} A}{\varepsilon_{r}}+B}{\frac{j K_{z 2}}{\varepsilon_{r}}+j K_{z i}}
$$

The magnitude of Eq.(12) shows the well known "Memory line" characteristics of the rough surface and it is related to $A_{d}$ in Eq.(12). The phase of $\mathrm{ACF} / \mathrm{FCF}$ is the phase of a complex number from contributions of five terms shown in Eq. (11). The first three terms in Eq.(11) are contributions from surface scattering. Each of these three terms can be written in the form of Eq.(12) where the phase dependent terms only reside $\Theta$ shown in Eq.(13). The last two terms in Eq. (11) are the contribution from the volume scattering explained in the next section. Note that the phase of $\mathrm{ACF} / \mathrm{FCF}$ of the surface scattering is independent of surface characteristics (rms height $\sigma$, correlation length $l$ ). However these surface characteristics dictate which surface is the dominant one in terms of total phase because, as shown in Eq.(12), the magnitude of $\mathrm{ACF} / \mathrm{FCF}$ returned to the receiver depends on the surface characteristics and the amount of wave incident upon each surface, which can be determined by coherent transmission line model explained previously.

\section{Incoherent component-volume scattering}

For volume scattering, we apply the Rayleigh scattering approximation because the particles are very small compared to the wavelength. We further assume that the volume scattering occur only in ice layer. The $\mathrm{ACF} / \mathrm{FCF}$ are given by

$$
\begin{aligned}
\left\langle\psi_{v}\left(\theta_{s 1}, \omega_{1}\right) \psi_{v}^{*}\left(\theta_{s 2}, \omega_{2}\right)\right\rangle & =\frac{f\left(\hat{o}_{1}, \hat{i}_{1}\right) f^{*}\left(\hat{o}_{2}, \hat{i}_{2}\right)}{(4 \pi)^{2} R_{1} R_{2}} \psi_{i 1} \psi_{i 2}^{*} \times \\
& \exp \left(-j k_{1} R_{1}+j k_{2} R_{2}\right) I
\end{aligned}
$$

where

$$
I=\rho V_{c} \exp \left(-\frac{v_{d x}^{2} L_{x}^{2}}{4}-\frac{v_{d y}^{2} L_{y}^{2}}{4}\right) \operatorname{sinc}\left(\frac{v_{z d} d}{2}\right) \exp \left[j\left(\mathbf{q}_{1}-\mathbf{q}_{2}\right) \cdot \mathbf{r}_{c}\right],
$$

$\rho$ is the particle density, $\mathbf{r}_{c}$ is the vector coordinate of the center of the volume, $V_{c}=L_{x} L_{y} d$ is the common volume. $\psi_{i 1}$ and $\psi_{i 2}$ are the coherent field incident at the top of ice layer for wave 1 and wave 2 , respectively. The scattering amplitude is $f(\hat{o}, \hat{i})=\frac{k^{2}}{4 \pi}\left|\frac{3\left(\varepsilon_{r}-1\right)}{\left(\varepsilon_{r}+2\right)}\right| V_{p} \sin \chi \quad$ where $V_{p}$ is the volume of the inclusions, $\chi$ is the angle between $\hat{o}$ and $\hat{e}$ (electrical field vector), $L_{x}$ and $L_{y}$ are the illumination length in the $x$-direction and $y$-direction, respectively,

$$
\begin{aligned}
\mathbf{q}_{1}= & k_{1}\left(\hat{i_{1}}-\hat{o}_{1}\right)=v_{1 x} \hat{x}+v_{1 y} \hat{y}+v_{1 z} \hat{z} \\
= & k_{1}\left[\sin \theta_{1 o} \cos \phi_{1 o}-\sin \theta_{1 i} \cos \phi_{1 i}\right] \hat{x} \\
& +k_{1}\left[\sin \theta_{1 o} \sin \phi_{1 o}-\sin \theta_{1 i} \sin \phi_{1 i}\right] \hat{y} \\
& +k_{1}\left[\cos \theta_{1 o}+\cos \theta_{1 i}\right] \hat{z}, \\
\mathbf{q}_{2}= & k_{2}\left(\hat{i}_{2}-\hat{o}_{2}\right)=v_{2 x} \hat{x}+v_{2 y} \hat{y}+v_{2 z} \hat{z} \\
= & k_{2}\left[\sin \theta_{2 o} \cos \phi_{2 o}-\sin \theta_{2 i} \cos \phi_{2 i}\right] \hat{x} \\
& +k_{2}\left[\sin \theta_{2 o} \sin \phi_{2 o}-\sin \theta_{2 i} \sin \phi_{2 i}\right] \hat{y} \\
& +k_{2}\left[\cos \theta_{2 o}+\cos \theta_{2 i}\right] \hat{z}, \quad v_{z d}=v_{1 z}-v_{2 z} . \text { The function } \\
v_{x d} & =v_{1 x}-v_{2 x} ; \quad v_{y d}=v_{1 y}-v_{2 y} ; \quad v \text { The }
\end{aligned}
$$

$I$ gives the "Memory dot" characteristic to the volume scattering correlation function. Note that the correlation functions for volume scattering are calculated based on threedimensional geometry for the spherical particles. On the other hand, the correlation functions for surface scattering explained in the previous section are based on two-dimensional geometry, which is not entirely matched. In our future work, we will include the correlation function for surface scattering based on three-dimensional geometry.

\section{ACF/FCF results}

We study the behavior of the ACF/FCF using the following parameters:

Wave 1: $137 \mathrm{MHz}$ center frequency with incident angle of 30 degree and observation angle at backscattering direction.

$\left(\theta_{1 i}=30^{\circ}, \theta_{1 o}=-30^{\circ}, f_{1}=137 \mathrm{MHz}\right)$

Wave 2: $162 \mathrm{MHz}$ center frequency with varying incident angle and observation angle. $\left(\theta_{2 i}=-\theta_{2 o}, f_{2}=162 \mathrm{MHz}\right)$

Layer and surface characteristics are listed in Table I at the snow depth of $8 \mathrm{~cm}$ and ice layer thickness of $2 \mathrm{~m}$. Here, we consider the brine inclusion with radius of $3 \mathrm{~mm}$ and fractional volume of 0.001 and the air bubbles with radius of 1 $\mathrm{mm}$ and fraction volume of 0.001 . The ACF/FCF values for each rough surfaces and volume scattering are normalized to the total ACF/FCF. The magnitude of the normalized $\mathrm{ACF} / \mathrm{FCF}$ as functions of the incident and observation angles of the second wave are shown in Fig. 3 which shows the memory line for the rough surface $\mathrm{ACF} / \mathrm{FCF}$ (left) and the memory dots for the volume scattering ACF/FCF (right). The dominant contribution is found to be from the ice-seawater (third) interface.

In order to extract information about the thickness of the ice, we need to obtain the sufficient amount of scattering from the rough surface 3 (ice-seawater interface). To maximize the desired surface scattering and to minimize the volume scattering effects, we can choose appropriate combinations of incident and observation angles for both waves as shown in Fig.3. 


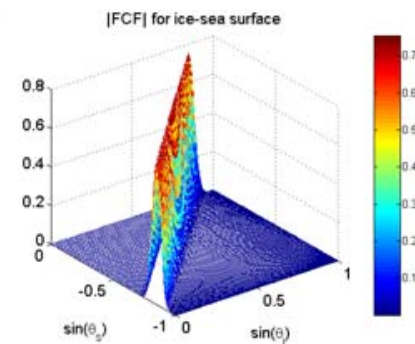

(A)

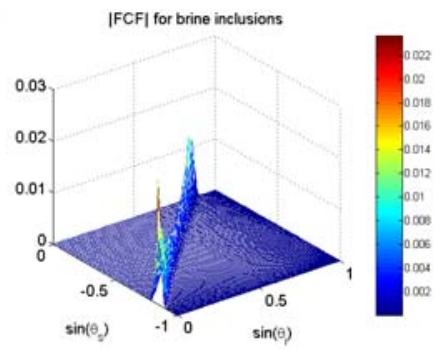

(B)
Fig 3. Correlation function (A) rough surface (surface 3) and (B) volume scattering (brine inclusion).

Fig. 4. shows the phase of the ACF/FCF as a function of the ice thickness. Here, We use correlation of wave one with frequency of $137 \mathrm{MHz}$, incident angle of 30 degree and observation angle in the back direction and wave two with frequency of $162 \mathrm{MHz}$, incident angle of 25 degree and observation angle in the back direction. These frequencies and incident and observation angles lies in the memory line and the effect from the volume scattering are mostly suppressed.
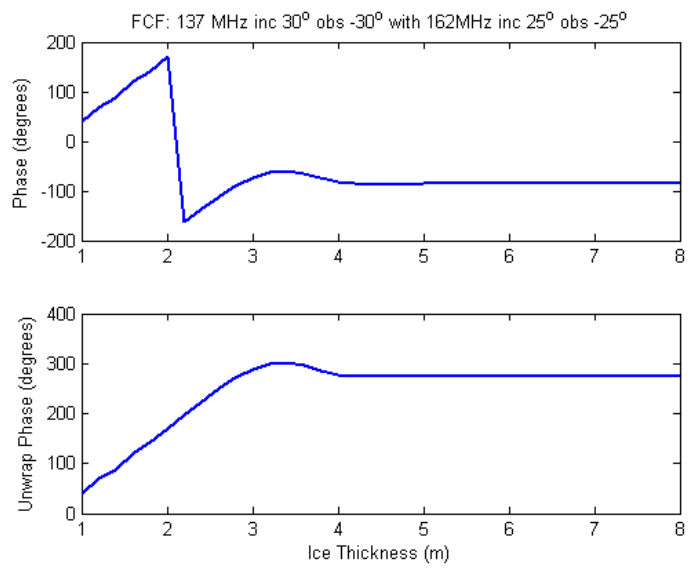

Fig 4. Phase of ACF/FCF as a function of ice thickness.

In this example, the phase of $\mathrm{ACF} / \mathrm{FCF}$ is linearly related to the ice thickness up to $3 \mathrm{~m}$. Beyond $3 \mathrm{~m}$, the phase is independent of ice thickness. This is due to the fact that the attenuation within a sea ice layer contributes significantly less signal return from the sea ice- water interface when the ice becomes thicker than $3 \mathrm{~m}$. According to our surfaces parameters, contribution from surface 2 (snow-ice) becomes dominant when ice thickness exceeds $3 \mathrm{~m}$. This limitation is strongly dependent on the characteristics of ice and the rough interface between each layer in our model.

\section{Sea ice Thickness Retrieval Algorithms}

From the previous section, the results suggest that the phase of FCF can be used to estimate the ice thickness. We apply several methods to estimate the ice thickness based on the measurement of phase of FCF. Here, we use simulated forward data to make the estimation.

First, we employ the gradient descent (GD) method to estimate the ice thickness. GD method is to minimize a cost function $\left(\psi(h)-\psi_{m}\right)^{2}$ where $\psi(h)$ is the phase of FCF calculated from the forward model. $\psi_{m}$ is the measured phase of FCF, which in this case, we use simulated data. Let $h_{o}$ be the first estimate of the thickness, we can improve the solution by

$h_{1}=h_{0}-2 \mu \frac{\partial \psi(h)}{\partial h}\left(\psi(h)-\psi_{m}\right)$

where $\mu$ is the iteration step. This process can be done iteratively until the solution converges within a prescribed margin. Since the phase of FCF $(\psi)$ is a complicated function of height $h$, we use the numerical derivative.

Second, we apply the least square error concept to the estimation. In the case where we have independent measurements of the $\mathrm{ACF} / \mathrm{FCF}$, either by changing combination of frequencies or angles or both, we can apply multiple values and form a minimization problem on the average error. In lease square (LSQ) method, we want to find the answer of the equation $\left(\psi_{m}-\psi\left(h_{s}\right)\right)^{2}=0$. Starting with an initial estimate $h_{o}$, we can find the increment $\Delta$ of the estimate to approach the solution. We have

$\psi_{m}-\psi\left(h_{0}+\Delta\right)=\psi_{m}-\left[\psi\left(h_{0}\right)+\left.\Delta \frac{\partial \psi}{\partial h}\right|_{h_{0}}\right]$.

For multiple data points, we can form a matrix equation.

The new estimation of the ice thickness is $h_{1}=h_{0}+\Delta$. This procedure can be iterated until the solution converges $\left(\left|h_{\text {new }}-h_{\text {old }}\right| \rightarrow \varepsilon\right)$.

Notice that both GD and LSQ method require a first estimate $h_{o}$. From the formulation of the small perturbation approximation explained in previous section, we can make a few assumptions to derive the first estimation. We assume that the contribution from the snow is small; therefore, we derive the phase of FCF in the case where snow thickness is zero. The expression for $h_{o}$ is not shown here due to the space limitations.

The performance of the GD and LSQ method are shown in Fig. 5. For GD method, we use wave 1 of $137 \mathrm{MHz}$ with incident angle of 30 degree and back direction observation and wave 2 of $162 \mathrm{MHz}$ with incident angle of 25 degree and back direction observation. For LSQ method we includes 7 data points using bandwidth of $6 \mathrm{MHz}$. The set of frequencies used for LSQ are listed in Table II. Measured data is the simulated data from forward model. The first estimations are calculated based on zero snow thickness, and then, the results are fed to the GD and LSQ algorithm. In this case, we assumed that the parameters used in the estimation, including the dielectric constants and snow depth, are known. As a result, the error of the estimation is very small.

Third, we employ a genetic algorithm (GA) optimization approach for sea-ice thickness estimation, $h$. For GA, we consider a fitness function or objective function for maximization within a constrained range of input parameters $h$. A binary coding for the parameters $h$ is used [7]. The fitness function is given in Eq (17), and we use it to assign a 
fitness value to each of the individuals, $h$, in the GA produce population. In equation (17), $\psi(h)$ is the phase of $\mathrm{FCF}$ calculated from the forward model for each population, and $\psi_{m}$ is the measured phase of FCF, which in this case, we use simulated data.

$$
\text { fitness }=e^{-\left(\psi_{m}-\psi(h)\right)^{2}}
$$

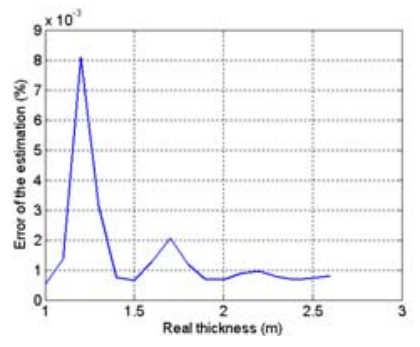

(A)

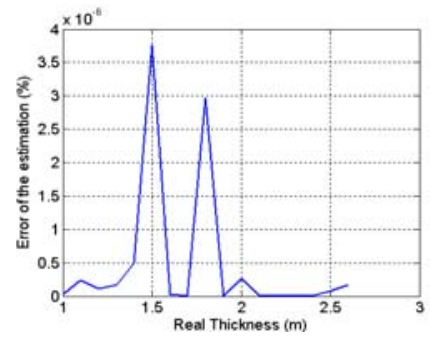

(B)
Fig 5. Error of the ice thickness estimation (\%) as a function of the true ice thickness for (A) GD method and (B) LSQ method.

Fig. 6a shows the GA estimated thickness results versus real thickness for a population of size 10 . The error in the estimation is given in Fig. 6b. For a particular trial (real thickness $h=0.75 \mathrm{~m}$ ) of this example, a fitness value of fitness $=0.998760$ was achieved after only 13 generations. A near optimal fitness value of fitness $=0.999999$ was achieved after 29 generations with the parameter $h=0.75 \mathrm{~m}$.

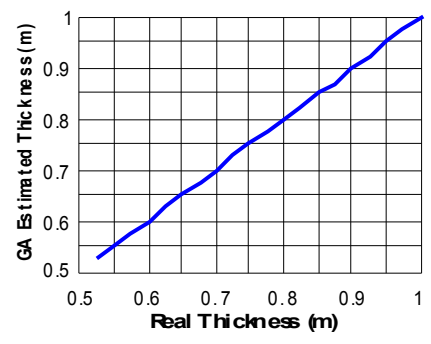

(A)

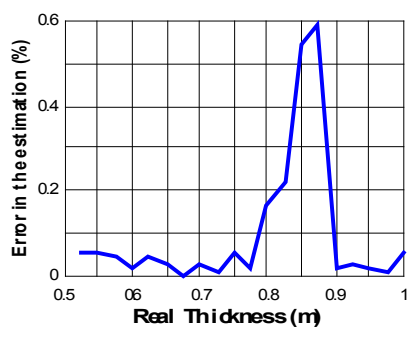

(B)
Fig. 6 (A) GA estimated thickness vs. real thickness and, (B) Error in the estimation as a function of real thickness.

Next, we investigate the cases where the other parameters are not completely known. First, we investigate the effect of uncertainty of dielectric constant of ice to the estimation. As the mixing formula (Eq.(1)) suggests, the volume fraction of brine inclusion dictates the dielectric constant of the bulk ice. We use the simulate data based on fractional volume of $5 \%$ but the estimation algorithms employ other value of fractional volume. The results are shown in Fig. 7A. It exhibits almost linear relationship between the deviation of the fractional volume to the true value and the percentage of error of the ice thickness estimation.

Second, we consider the error in snow depth parameter. The true snow depth is $8 \mathrm{~cm}$. We calculate the case when the assumed snow depth changes from no snow to $30 \mathrm{~cm}$. The results are shown in Fig. 7B. The error of ice thickness estimation is shown as a function of the assumed snow depth at several true ice thickness values. It indicates that the snow depth is also an important parameter for accurately estimate the ice thickness. The results also suggest that the larger the ice thickness the smaller the effect of snow on the estimation.

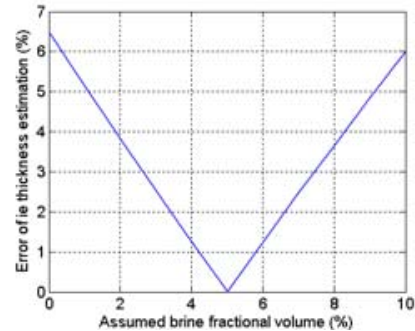

(A)

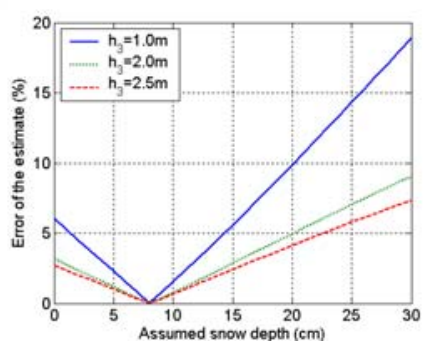

(B)
Fig. 7 (A) Relationship of assumed brine fractional volume and the error estimation of the ice thickness (true brine fractional volume is $5 \%$ ). (B) The error of ice thickness estimation as a function of the assumed snow depth by GD method. Here, the true snow depth is $8 \mathrm{~cm}$.

\section{CONCLUSION}

Sea ice thickness retrieval can be done efficiently by $\mathrm{ACF} / \mathrm{FCF}$ method. We used several methods in the inversion calculations namely gradient descent (GD), least square (LSQ) method, and genetic algorithm (GA). Good agreement is shown between GA, GD and LSQ results, when a single unknown variable - sea-ice thickness - is to be determined. Our forward calculations use four-layer sea-ice model (air, snow, sea-ice, and seawater). The model developed here to obtain the $\mathrm{ACF} / \mathrm{FCF}$ functions is based on small perturbation theory. We used the ACF/FCF functions to gain insight into coherence amplitude and phase sensitivity with ice depth, surface layers roughness, and volume scattering suppression. The effect of snow cover and the knowledge of the dielectric constant of the brine inclusions on ice thickness retrieval are discussed. We are currently developing a VHF and Ku-band (for snow cover characteristics) field experimental radar to validate and improve the inversion model and its prediction through a sea ice field experiment conducted on the Arctic Ocean ice cover.

\section{REFERENCES}

[1] A. Ishimaru, Wave Propagation and Scattering in Random Media. Pistacaway, NJ: IEEE Press, 1997.

[2] A. Ishimaru, Electromagnetic Wave Propagation, Radiation, and Scattering, Englewood Cilff, NJ: Prentice Hall, 1991.

[3] F. T. Ulaby, R. K. Moore, and A. K. Fung, Microwave Remote Sensing: Active and Passive, Vol. 3, Norwood, MA: Artech House, 1990.

[4] Y. Kuga, C. T. C. Le, A. Ishimaru, L. Ailes-Sengers, "Analytical, experimental, and numerical studies of angular memory signatures of waves scattered from one-dimensional rough surfaces," IEEE Transactions on Geoscience and Remote Sensing, Vol. 34, No. 6, pp. 1300-7, 1996.

[5] A. K. Fung, Microwave Scattering and Emission Models and Their Applications, Norwood, MA: Artech House, 1994.

[6] C. T. C. Le, "Angular memory effect and its interferometric applications in rough surface mean height profiling," Ph. D. thesis, Dept. Electron. Eng., Univ. of Washington, Seattle, WA, USA, 1996.

[7] D. E. Goldberg, Genetic Algorithms in Search, Optimization and Machine Learning. Addison-Wesley, Reading, MA, 1989. 